“C 2010 IEEE. Personal use of this material is permitted. Permission from IEEE must be obtained for all other uses, in any current or future media, including reprinting/republishing this material for advertising or promotional purposes, creating new collective works, for resale or redistribution to servers or lists, or reuse of any copyrighted component of this work in other works.” 


\title{
Initial Rotor Position and Magnetic Polarity Identification of PM Synchronous Machine based on Nonlinear Machine Model and Finite Element Analysis
}

\author{
Yi Wang ${ }^{1}$, Ningning Guo ${ }^{2}$, Jianguo Zhu ${ }^{1}$, Senior Member, IEEE, Nana Duan ${ }^{2}$, Shuhong Wang ${ }^{2}$, \\ Youguang Guo ${ }^{1}$, Senior Member, IEEE, Wei Xu${ }^{1}$, Yongjian $\mathrm{Li}^{1}$ \\ ${ }^{1}$ School of Electrical, Mechanical and Mechatronic Systems, University of Technology, Sydney, NSW 2007, Australia \\ ${ }^{2}$ Faculty of Electrical Engineering, Xi'an Jiaotong University, Xi'an 710049, China
}

In this paper, a DC voltage pulse injection based method is proposed to detect the initial position and rotor polarity of permanent magnet synchronous motor (PMSM). The rotor angle vibration is expressed analytically as a function of the injected pulse. The nonlinear numerical simulation model of PMSM is employed, in which the saturation saliency is incorporated as well as the structural saliency. The proposed scheme is simulated and verified by using the nonlinear model and a two-dimensional finite element method (FEM) algorithm, respectively. The initial position detection is tested at different starting positions and the results show that the proposed scheme can be used to identify the PMSM initial rotor position.

Index Terms - Finite element magnetic field analysis, initial rotor position, nonlinear magnetic model, permanent magnet (PM) machine.

\section{INTRODUCTION}

$\mathrm{T}$ HE PERMANENT magnet synchronous machines (PMSMs) have found wide applications. However, a rotor position sensor is often required in a PMSM drive system, which will not only increase the system cost, but also perhaps more importantly reduce the system reliability [1]. Even for some instinctive sensorless drive methods such as the direct torque control (DTC), the initial rotor position detection is unavoidable [2].

A number of signal injection techniques for position sensorless and initial rotor position detection have been proposed based on tracking the rotor magnetic saliency [3]. The saturation saliency of PMSM has to be utilized for identifying the rotor polarity of PMSM. However, the saturation saliency is not incorporated in the traditional linear machine model. A nonlinear numerical PMSM model has been developed in [4], in which both the structural and the saturation saliencies are incorporated. The simulation and verification of the proposed sensorless drive schemes could be carried out instead of using the experimental trial and error method.

In [5], a DC pulse injection method was proposed and implemented, but the rotor vibration during the signal injection was not discussed. A numerical simulation was carried out by Yan et al. in [6]. However, the rotor position is fixed during the injection and the nonlinear model reported is only based on 5.5 A current offset, which is not enough to analyze the current response in the stator windings.

In this paper, an initial rotor position detection method is proposed based on DC pulse injection. The rotor angle vibration is expressed as a response function of the injected signal. A series of DC voltage pulses are injected into the stator windings to determine the rotor initial position. To minimize the rotor vibration, the rotor axis position is detected firstly by injecting low magnitude pulses. Two high magnitude voltage pulses are then injected to detect the rotor polarity based on the saturation effect. The nonlinear machine model and a two-dimensional (2D) finite element method (FEM) algorithm are applied to verify and simulate the proposed scheme. The initial rotor position detection performance is evaluated at different initial rotor angles. The results show that the proposed scheme is suitable for SPMSM initial position and magnet polarity detection.

\section{NONLINEAR MODEL OF SPMSM WITH SALIENCIES}

In an SPMSM, the observable total flux linkage $\lambda_{t}$ inside the air-gap is contributed by both the stator currents and the permanent magnets on the rotor and it is the link between the stator and rotor magnetic fields. The three-phase flux linkages $\lambda_{a b c}$ are defined as the projection of $\lambda_{t}$ on the stator reference frame. The voltage equation of the stator windings can be written as

$$
v_{a b c}=R_{s} i_{a b c}+\frac{d}{d t} \lambda_{a b c}
$$

where $v_{a b c}, i_{a b c}$ and $R_{s}$ are the phase voltages, currents and winding resistance in the stator reference frame, respectively.

In a nonlinear model, the magnetic saturation should be considered and the inductances are expressed as incremental inductances, which vary against the stator current. Then a composite function can be used to express the flux linkage as

$$
\lambda_{a}=f\left(i_{a b c}, \theta\right)
$$

where $\theta$ is the rotor position angle.

The following differential equations can be obtained

$$
\left[\begin{array}{c}
v_{a} \\
v_{b} \\
v_{c}
\end{array}\right]=R_{s}\left[\begin{array}{c}
i_{a} \\
i_{b} \\
i_{c}
\end{array}\right]+L_{a b c}^{*} \frac{d}{d t}\left[\begin{array}{c}
i_{a} \\
i_{b} \\
i_{c}
\end{array}\right]+\frac{\partial}{\partial \theta}\left[\begin{array}{c}
\lambda_{a} \\
\lambda_{b} \\
\lambda_{c}
\end{array}\right] \omega_{e}
$$


where $\frac{\partial}{\partial \theta}\left[\begin{array}{l}\lambda_{a} \\ \lambda_{b} \\ \lambda_{c}\end{array}\right] \omega_{e}$ is the rotating back-emf in the threephase windings;

$$
L_{a b c}^{*}=\left[\begin{array}{ccc}
L_{a a}^{*}\left(i_{a b c}, \theta\right) & L_{a b}^{*}\left(i_{a b c}, \theta\right) & L_{a c}^{*}\left(i_{a b c}, \theta\right) \\
L_{b a}^{*}\left(i_{a b c}, \theta\right) & L_{b b}^{*}\left(i_{a b c}, \theta\right) & L_{b c}^{*}\left(i_{a b c}, \theta\right) \\
L_{c a}^{*}\left(i_{a b c}, \theta\right) & L_{c b}^{*}\left(i_{a b c}, \theta\right) & L_{c c}^{*}\left(i_{a b c}, \theta\right)
\end{array}\right] \text { is the }
$$

nonlinear inductance matrix which is also a composite function of the stator currents and rotor position.

A nonlinear model of PMSM is built up incorporating the saturation saliency, based on which a MATLAB/SIMULINK model is carried out to simulate the PMSM drive schemes [4].

\section{INITIAL ROTOR POSITION DETECTION}

\section{A. Rotor Vibration Limitation}

Most of the reported initial rotor position detection methods are based on electrical excitation and the injected signal should be carefully chosen in order to keep the rotor stationary.

In the proposed pulse injection method, a series of DC voltage pulses are injected into the stator winding to identify the rotor position. Once the pulses are applied to detect the rotor initial position, the resultant rotor speed is small enough so that the friction could be neglected. On the other hand, the angle vibrating limitation calculated under the zero friction assumption could easily satisfy the real system, where the unavoidable friction will further limit the rotor movement. Then the rotor mechanical angle can be solved as

$$
\theta=\delta \frac{2}{p}+\frac{p}{2} \frac{\lambda_{m} V_{i n} \sin \delta}{J R}\left[\frac{t^{2}}{2}+\left(\frac{L_{q}}{R}\right)^{2}\left(1-e^{-\frac{R}{L_{q}} t}\right)-\frac{L_{q}}{R} t\right]
$$

where $\delta$ is the electrical angle between the rotor d-axis and the injected voltage vector, $V_{i n}$ is magnitude of the injected voltage pulse, and $J$ is the mechanical inertia.

For any initial rotor position, the maximum rotor angle vibration under the DC injected voltage pulse can be expressed as

$$
\Delta \theta= \pm \frac{p}{2} \frac{\lambda_{m} V_{i n}}{J R}\left[\frac{\Delta t^{2}}{2}+\left(\frac{L_{q}}{R}\right)^{2}\left(1-e^{-\frac{R}{L_{q}} \Delta t}\right)-\frac{L_{q}}{R} \Delta t\right]
$$

where $\Delta t$ is the injected pulse width.

\section{B. DC Pulse Injection}

One positive unit of the injection pulses is shown in Fig. 1. A positive voltage pulse is firstly applied and the increasing phase current response is shown in Fig. 1. Then a negative pulse is injected right after the positive pulse to bring down the phase current back to zero and prevent the rotor from moving. The peak current value will be recorded, which is inversely proportional to the phase inductance. In the negative pulse unit, a negative DC pulse will be applied and a negative peak current value will be recorded.
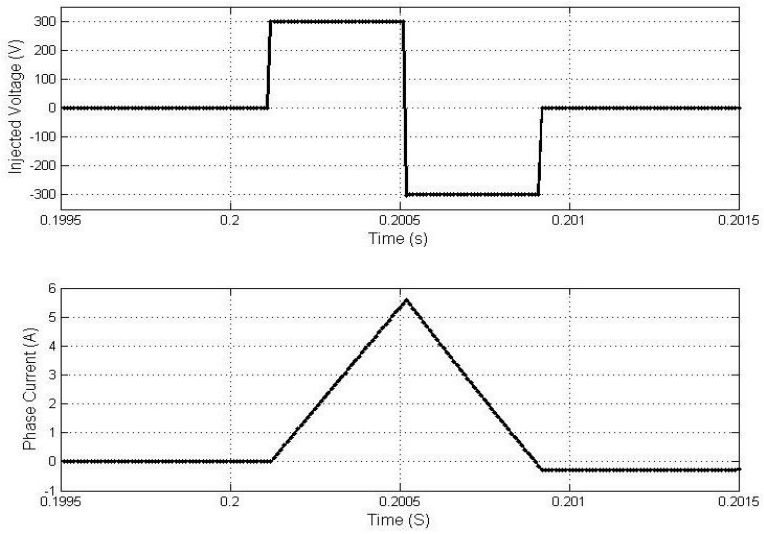

Fig. 1. Example of injected DC voltage pulses and excited phase current.

In order to limit the rotor vibration, the injected voltage pulses are chosen based on (5) and the pulse width is set as $0.0004 \mathrm{~s}$. When a low voltage pulse, $50 \mathrm{~V}$, is injected, the rotor movement is \pm 0.08 electrical degree. When a high voltage pulse, $300 \mathrm{~V}$, is injected, the rotor movement is \pm 0.5 electrical degree. Fig. 2 shows the maximum rotor vibrating angle under different voltage pulses injection.

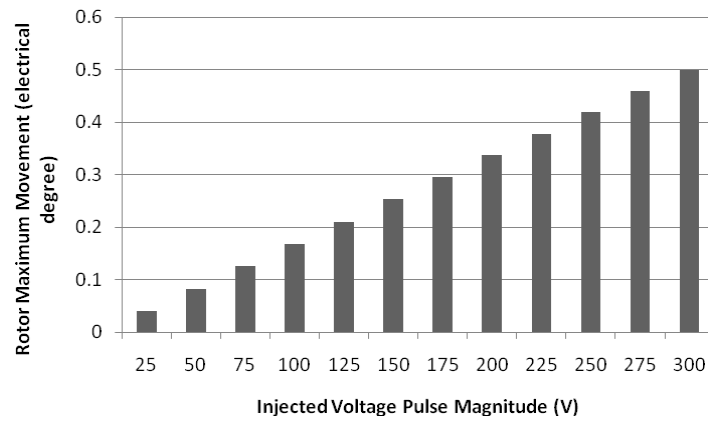

Fig. 2. Maximum rotor movement under voltage pulses, $\Delta t=0.0004 \mathrm{~s}$.

The modeled machine, which has six poles, can be calculated from Fig. 2 that the maximum rotor vibrating angle of $300 \mathrm{~V}$ pulse injection is smaller than 0.2 mechanical degrees. This value is acceptable for the numerical simulation to assume the rotor is kept at standstill. As discussed above, this angle value will be further reduced in the real time experiment, where the shaft friction does exist.

As mentioned above, the recorded peak current value is inversely proportional to the phase self-inductance, which is a periodical function of the rotor angle. In Fig. 3, the rotor initial position is set at different values and DC pluses are injected. The recorded peak current values against the initial rotor angle are shown in Fig. 3, where the injected voltage pulse is set to $50 \mathrm{~V}$ and $300 \mathrm{~V}$, respectively.

As shown in Fig. 3 (a), the peak current values of N- and Spole are nearly equal under low voltage pulses excitation. When the injected voltage magnitude is increased to a higher value, which will induce a larger current in the stator phase, the stator core will be saturated more and the peak current value difference between $\mathrm{N}$ - or S-pole will increase to a higher 
level as shown in Fig. 3 (b). This current difference is collected to identify the rotor polarity. Fig. 4 shows the peak current difference when the $\mathrm{N}$ - or S-pole aligns with the under-injection stator phase. It can be found out that the relationship between the injected voltage magnitude and the current difference is not linear because of the nonlinear inductance model.

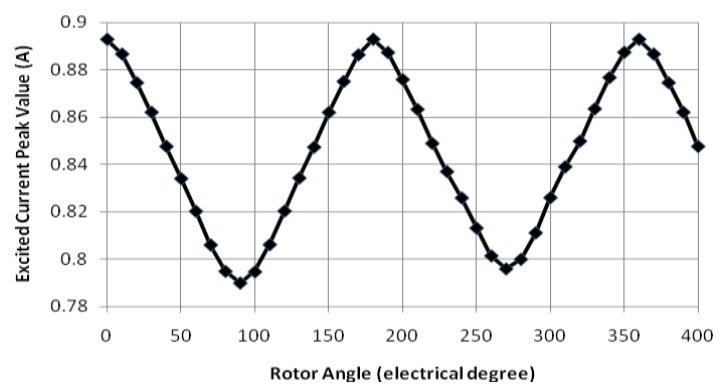

(a)

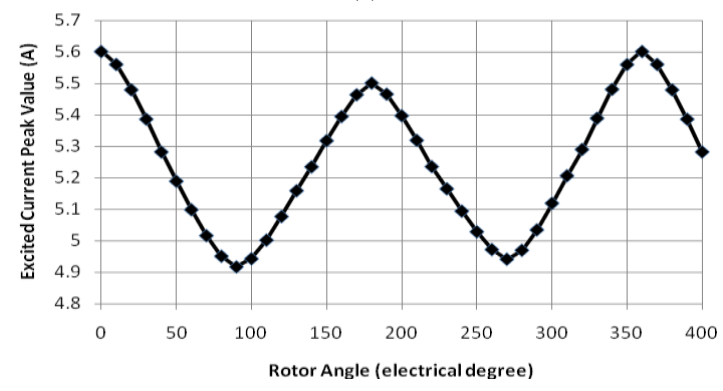

(b)

Fig. 3. Excited peak current values against rotor position angle: (a). Peak current values under low voltage pulse excitation, (b). Peak current values under high voltage pulse excitation.

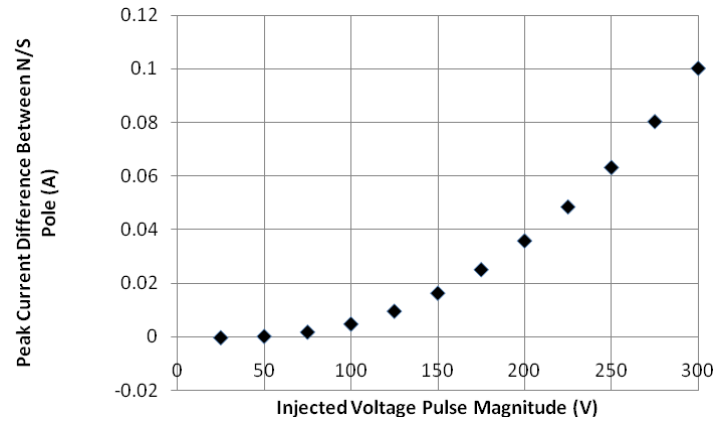

Fig. 4. N/S pole excited peak current differences under different voltage pulses injection, $\Delta t=0.0004 \mathrm{~s}$.

\section{Initial Rotor Position Detection}

There are two steps to obtain the rotor position. Low and medium magnitude pulses are injected firstly to estimate the rotor axis position and then higher magnitude pulses are used to identify the rotor polarity.

For an unknown rotor position, a positive and a negative pulse unit are applied to stator phase, $A, B, C$, respectively. The response peak current values are marked as $I_{A+}, I_{A-}, I_{B+}, I_{B-}$ , $I_{C+}$ and $I_{C-}$, where $A, B, C$ represent the three stator phases and $+/$ - represent the injected positive or negative voltage pulse. The peak current values collected at different rotor angles can be approximately expressed as balanced threephase sinusoidal waves, as shown in Fig. 3 (a). In order to minimize the rotor movement, the low voltage pulses are applied firstly. The two largest current peak values in one phase, under positive and negative excitations, are used to determine the rotor axis position. As shown in Fig. 5, one electrical cycle is divided into six sectors and each sector can be addressed by tracking the maximum current peak value. For example, when the rotor d-axis is in sector 1 or 4 , the recorded phase $B$ peak current values, $I_{B+}$ and $I_{B-}$ will have larger magnitude. Then, the other two phases current peak values, $I_{A+}, I_{A-}, I_{C_{+}}$and $I_{C_{-}}$are picked up to calculate the angle offset inside the sector. Additionally, a set of medium magnitude voltage pulses is applied to phase $A$ and $C$ to minimize the calculation error of the offset angle. Therefore, the rotor axis position could be obtained in sector 1 and 4 .

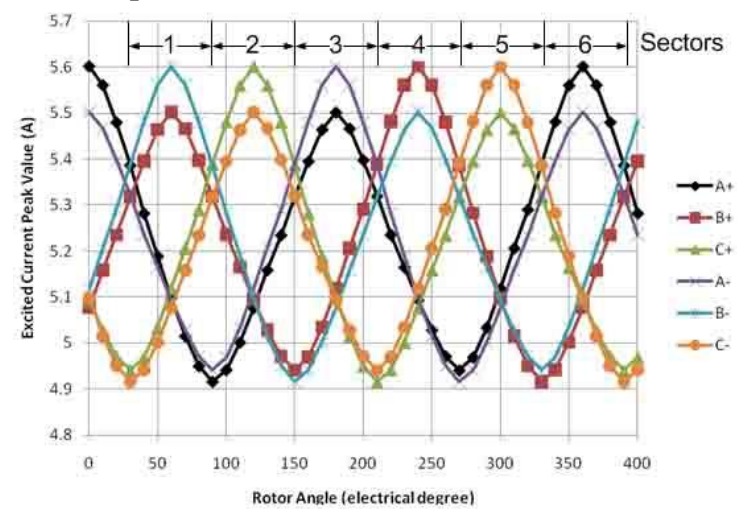

Fig. 5. Three-phase excited peak current under high voltage pulses. $\left(V_{\text {in }}=300 \mathrm{~V}\right)$.

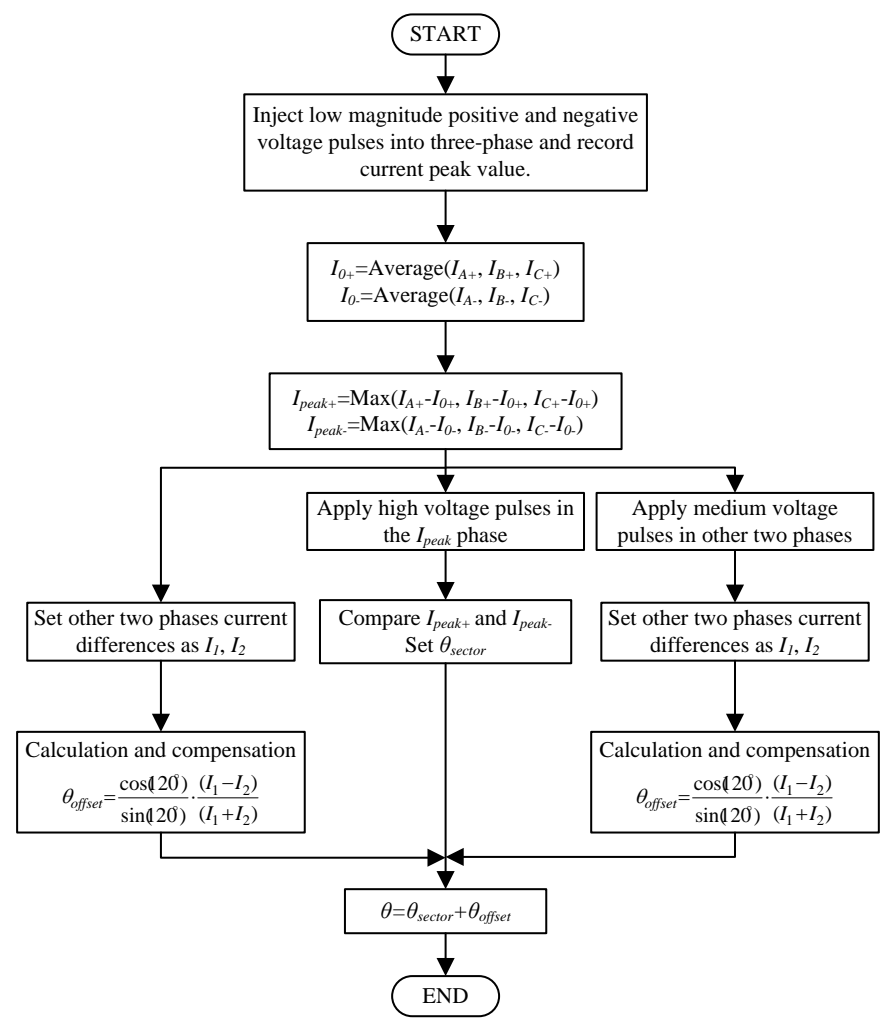

Fig. 6. Rotor position estimation procedure.

Then high magnitude positive and negative voltage pulses are then applied to phase $B$, which is close to the rotor d-axis. The peak current difference under the positive and negative 
pulses will be observable to detect the rotor polarity as shown in Fig. 5. After obtaining the rotor $\mathrm{N}$ pole sector number, the initial rotor angle can be determined as shown in Fig. 6. In this procedure, the high voltage pulses are only injected to the phase close to the d-axis so that the rotor vibration is minimized.

\section{FEM based calculation}

In order to verify the proposed nonlinear model, the 2D FEM is applied to numerically calculate the magnetic field of the machine. Fig. 7 shows the magnetic field distribution, where the DC voltage pulse is injected in stator phase $A$.

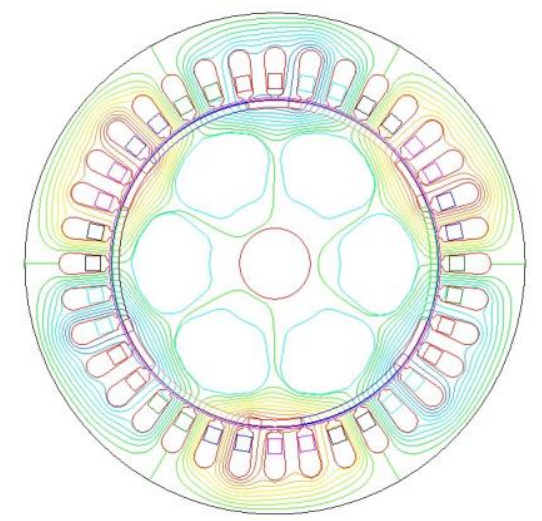

Fig. 7. Magnetic field distribution when voltage pulse is added in phase $A$.
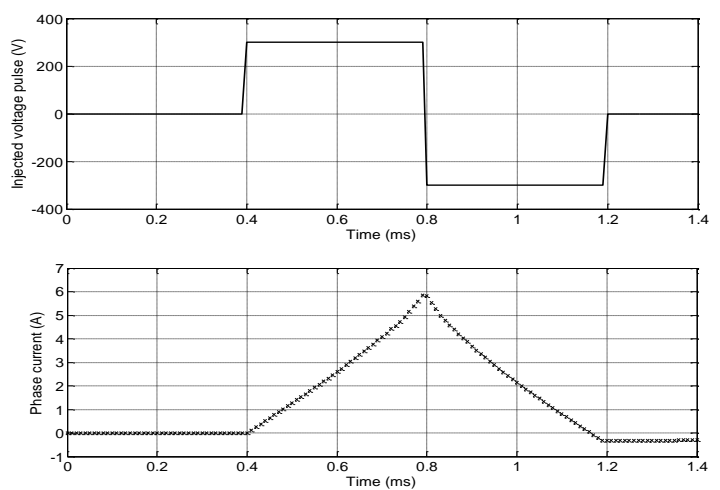

Fig. 8. Injected DC voltage pulse and calculated phase current.

Fig. 8 shows the injected positive unit of voltage pulses and the calculated phase current. It can be found that the calculated current responses coincide with the estimated values as in Fig. 1. The errors shown in Fig. 8 mainly come from the stator skewed slots.

\section{Simulation Performance}

The proposed method is applied to the nonlinear machine model in SIMULINK. The initial rotor position is set to several values and simulation is conducted. Fig. 9 shows the rotor angle detection results at different rotor positions. It can be found out that the estimated initial rotor angle is close to the real rotor position and the error of the estimation is limited within \pm 7 electrical degrees, or \pm 2.3 mechanical degrees. When the rotor position is away from the sector center, the error becomes bigger, because the three-phase peak current response is not exactly a second-order sinusoid of rotor position. At the same time, the rotor vibration is further reduced to $\pm 0.3 \%$, which is smaller than that in Fig. 2, because only the low and medium voltage pulses are injected to all the three phases and the high voltage pulses are injected only around d-axis so that the generated instantaneous torque is small.

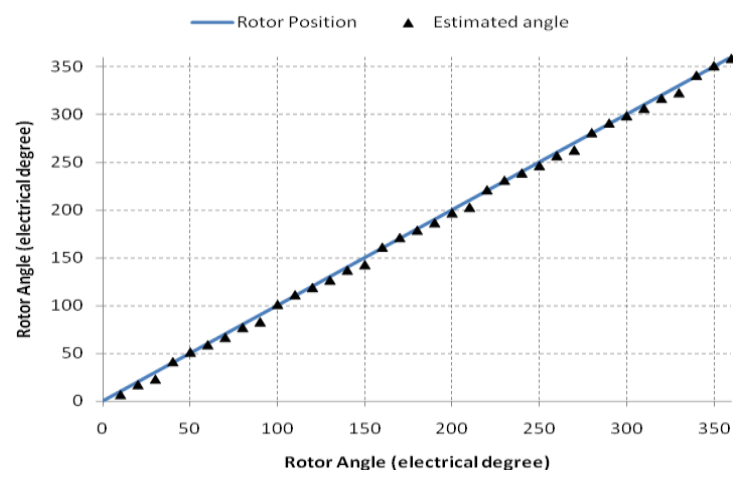

Fig. 9. Estimated initial rotor position.

\section{CONCLUSION}

In this paper, a DC pulse injection based method is proposed to detect the initial rotor position of PMSM. The rotor vibration analysis is carried out based on DC pulse injection, which is an effective initial rotor position detection scheme. The rotor maximum vibrating angle is expressed as a function of the width and magnitude of the injected pulse. The injected voltage signal is then specified to try to keep the rotor at standstill. Based on the injected DC pulses and the comprehensive nonlinear machine model, numerical simulation of the current response is carried out, in which the saturation saliency is detected and utilized to identify the rotor polarity. At the same time, an FEM algorithm is carried out to numerically calculate the magnetic field of the machine and verify the proposed scheme. The simulation and calculation are taken at several different initial rotor positions and the results show that the estimation error is small, showing that the proposed method can be applied to detect the initial rotor position and magnet polarity.

\section{REFERENCES}

[1] Y. Yamamoto, Y. Yoshida, and T. Ashikaga, "Sensorless control of PM motor using full order flux observer," IEEE Trans. Ind. Appl., vol. 124, no. 8, pp. 743-749, Aug. 2004.

[2] M. E. Haque, L. Zhong, and M. F. Rahman, "A sensorless initial rotor position estimation scheme for a direct torque controlled interior permanent magnet synchronous motor drive," IEEE Trans. Power Electron., vol. 18, no. 6, pp. 1376-1383, Nov. 2003.

[3] E. Robeischl, M. Schroedl, and M. Krammer, "Position-sensorless biaxial position control with industrial PM motor drives based on INFORM-method and back EMF model," in Proc. IEEE-IES Апnи. Conf., 5-8 Nov. 2002, vol. 1, pp. 668-673.

[4] Y. Wang, J. G. Zhu, S. H. Wang, Y. G. Guo, and W. Xu, "Nonlinear magnetic model of surface mounted PM machines incorporating saturation saliency," IEEE Trans. Magn., vol. 45, no. 10, pp. 4684-4687, Oct. 2009.

[5] P. B. Schmidt, "Method and apparatus for rotor angle detection," U.S. Patent 6172 498, Jan. 9, 2001.

[6] Y. Yan, J. G. Zhu and Y. G. Guo, "A direct torque controlled surface mounted PMSM drive with initial rotor position estimation based on structural and saturation saliencies," in Proc. IEEE IAS Annu. Meeting, New Orleans, LA, Sept. 2007, pp. 683-689. 